\title{
Coenzyme Q10 - gamma cyclodextrin complex is a powerful nutraceutical for anti-aging and health improvements
}

\author{
Yukiko Uekaji $^{*}$ and Keiji Terao ${ }^{1,2}$ \\ ${ }^{1}$ CycloChem Bio Co., Ltd., Japan \\ ${ }^{2}$ Graduate School of Medicine, Kobe University, Japan
}

\begin{abstract}
Coenzyme Q10 (CoQ10) is a fat-soluble, vitamin-like, benzoquinone compound that functions primarily as an antioxidant, as a membrane stabilizer and as a cofactor in the production of adenosine triphosphate (ATP) by oxidative phosphorylation. The solubility of CoQ10 in water is extremely low, resulting in a low bioavailability by oral administration. Therefore, we attempted to improve the low dissolution and bioavailability of CoQ10 by means of complexation with various cyclodextrins (CDs). We found that the bioavailability of CoQ10 could be significantly enhanced by complexation with CDs, especially by the use of $\gamma \mathrm{CD}$. The CoQ10 - $\gamma \mathrm{CD}$ complex reveals excellent pharmacokinetic properties not only significantly higher area under the CoQ10 concentration curve (AUC) in blood plasma from time $0-48 \mathrm{hr}$ and higher maximum plasma concentration $\left(\mathrm{C}_{\max }\right)$, but also the mean plasma levels even after 24 and 48 hours tended to be significantly higher after CoQ10 - $\gamma \mathrm{CD}$ complex administration to healthy adult volunteers when compared with CoQ10 formulated in microcrystalline cellulose and administrated in the same way as the complex. We found that this long-lasting high CoQ10 concentration in plasma provides various health benefits. The proven benefits are: 1 ) Curative effects on damaged human skins by aging, UV and other pollutants in the air, 2) Anti-oxidant activity (study on the reduction of 8-hydroxy-2-deoxy guanosine (8-OHdG) in urine), 3) Protection of muscle cells (study on the reduction of myogenic enzymes, creatinine phosphokinase (CPK) and lactate dehydrogenase (LDH)).
\end{abstract}

\section{Introduction}

Coenzyme Q10 (CoQ10) is a fat-soluble, vitamin-like, benzoquinone compound that functions primarily as an antioxidant, as a membrane stabilizer and as a cofactor in the production of adenosine triphosphate (ATP) by oxidative phosphorylation [1]. It has also been shown that it helps to preserve myocardial sodium-potassium ATPase activity and to stabilize myocardial calcium-dependent ion channels [2]. Due to its lipophilic property, CoQ10 is not well absorbed when taken orally by human beings. Therefore, formulations that could enhance the bioavailability of CoQ10 are desired.

The solubilisation of new drugs with poor solubility in water is essential for the pharmacological evaluation and drug development. There are various methods for improvement of aqueous solubility of lipophilic compounds in the nutraceutical and pharmaceutical fields: amorphous form, grinding, solid dispersion, micelle and inclusion complex [3,4].

Among them, the use of cyclodextrins (CDs) is well known. $\alpha, \beta$ or $\gamma \mathrm{CD}$ are widely used natural CDs, consisting of six, seven and eight D-glucopyranose residues, respectively, linked by $\alpha-1,4$ glycosidic bonds into a macrocycle. CDs can generally form inclusion complexes with various lipophilic substances as guests and thus have been utilized for improving water solubility, stability and bioavailability of those substances $[5,6]$.

We have reported that the bioavailability of CoQ10 could be significantly enhanced by the complexation with $\gamma \mathrm{CD}$ among natural $\mathrm{CDs}$ for the use in nutrition applications [7-11]. Interestingly, the CoQ10 - $\gamma \mathrm{CD}$ complex gives not only significantly higher area under the plasma CoQ10 concentration curve (AUC) for 0-48 hr and maximum plasma concentration $\left(\mathrm{C}_{\max }\right)$, but also the mean plasma levels at 24 and 48 hours tended to be significantly higher after CoQ10 - $\gamma$ CD complex administration to healthy adult volunteers when compared with CoQ10 formulated in microcrystalline cellulose and administrated in the same way as the complex.

The high dispersibility of CoQ10 by complexation with $\gamma \mathrm{CD}$ and the sustained release of CoQ10 from the complex are considered to provide the interesting effect of the long-lasting high CoQ10 concentration in plasma. After transfer from plasma to tissues CoQ10 can deliver various valuable anti-aging and health benefits. This article focuses on the proven benefits obtained from our recent analyses by the oral intake of CoQ10 - $\gamma \mathrm{CD}$ complex. These are: 1) Curative effect on damaged human skins by aging, UV and other aerial pollution, 2) Anti-oxidant activity (study on the reduction of 8-hydroxy-2-deoxyguanosine (8OHdG) in urine), 3) Protection of muscle cells (study on the reduction of myogenic enzymes, creatinine phosphokinase (CPK) and lactate dehydrogenase (LDH)).

Correspondence to: Yukiko Uekaji, CycloChem Bio Co., Ltd., 7-4-5 Minatojimaminamimachi, Chuo-ku, Kobe 650-0047 Japan, Tel: +81-(0)78-302-7073, Fax: +81-(0)78-302-7220, E-mail: yukiko.uekaji@cyclochem.com

Key words: coenzyme Q10, cyclodextrin, complexation, nutraceutical, bioavailability, anti-aging, antioxidant, membrane stabilizer, adenosine triphosphate production cofactor

Received: November 11, 2016; Accepted: December 29, 2016; Published: January 03, 2017 


\section{Materials and methods}

Materials: The ingredients and contents in the capsule are shown in Table 1. CoQ10 - $\gamma$ CD complex used is commercially available from Wacker Chemie AG, (Germany). Since CoQ10 is a photosensitive material, the complex was encapsulated in dark brown capsules.

Subjects: The study was performed in accordance with the requirements of the World Medical Association Declaration of Helsinki regarding protection of rights and welfare of human subjects participating in this study. The protocol was carefully explained to the volunteers and their written informed consents were prepared. Subjects were excluded based on the following conditions: 1) failure to receive drugs or daily food supplements known to affect or with the potential to affect this test, 2) having a history of allergic reactions attributed by compounds of similar composition to the test materials used in this study, 3) having a habit of drinking a lot of alcohol drink, 4) a pregnant, 5) a patient of hepatitis, hepatopathy, renal damage, myocardial infarction.

Screening: Statistically, it is known that the addicted cigarette smokers have more severe damages on skin and body tissue conditions than non-smokers [12]. To demonstrate the improvements and recoveries of various damaged conditions by the oral intake of CoQ10 - $\gamma \mathrm{CD}$ complex, 35 Japanese volunteers (ages: 30 - 60) consuming cigarettes on a daily basis (10 - 20 cigarettes a day) were recruited and they accepted the explanation of the meaning of this test. Further, 18 subjects were selected in order of lower skin elasticity as a basic index for damaged skin condition among the 35 smokers (Table 2).

Schedule: The subjects took daily three capsules of test materials with $200 \mathrm{~mL}$ of lukewarm water for six weeks. The dose of CoQ10 is adjusted at $30 \mathrm{mg} /$ day. The required data for each study were obtained for three times during the test period before intake, after intake for three weeks and six weeks (Figure 1).

\section{Study on the curative effect on damaged human skins}

The morphological changes of skin mainly are caused by aging and external stresses like ultraviolet (UV) radiation and reactive oxygen species. CoQ10 is known to play an important role as an antioxidant on human keratinocytes and to stimulate collagen production by human fibroblasts in corium to be extremely useful for the improvements and recoveries of various damaged skin conditions [13]. However, there are few clinical studies to verify the efficacy of oral intake of CoQ10 for damaged and aged skin. Hoppe et al. showed that topical application of CoQ10 for three months reduced the wrinkles around the eyes in humans [14]. But, to our knowledge, there has been no detailed study available so far for oral supplementation of CoQ10 applied for damaged or aged skins. The visible signs of aging human skin are not only amplified wrinkle formation, but also decreased texture, loss of elasticity, loss of moisture, loss of thickness and increased roughness

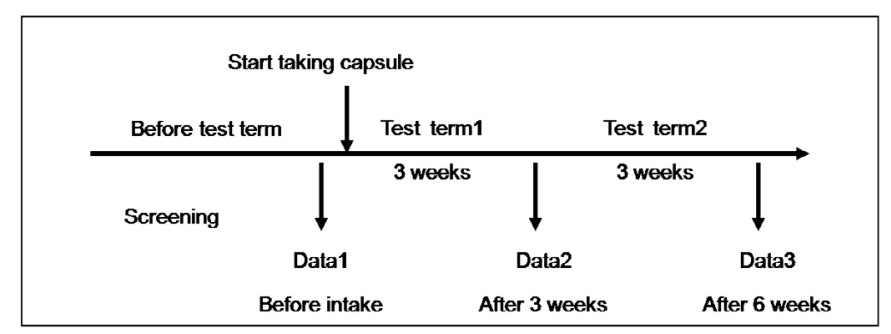

Figure 1. Schedule. of skin [15]. Hence, we evaluated the curative effects on damaged human skins with regard to the changes of wrinkle, texture, elasticity and moisture content by the oral intake of CoQ10 - $\gamma \mathrm{CD}$ complex with daily dose of CoQ10 at $30 \mathrm{mg}$.

Test procedure: The subjects washed their faces with their own facial cleansing cream at the facility of Institute of General Health Development Co., Ltd., Tokyo, Japan. For the sake of having equal conditions among subjects, they stayed at the air conditioning room $\left(22^{\circ} \mathrm{C} \pm 2{ }^{\circ} \mathrm{C}\right.$, relative humidity $\left.(\mathrm{RH}) 50 \% \pm 15 \%\right)$ for 25 minutes, and then, stayed at the room $\left(22^{\circ} \mathrm{C} \pm 1{ }^{\circ} \mathrm{C}, \mathrm{RH} 50 \% \pm 10 \%\right)$ for 5 minutes just before the evaluations.

\section{Study on antioxidant effect by the evaluation of 8-OHdG in urine}

Oxidative stress is thought to play a major role in carcinogenesis $[16,17]$, and increased levels of 8-hydroxydeoxyguanosine (8-OHdG) are detected in urine of smokers. The source of urinary 8-OHdG may be the hydrolysis of 8-OHdGTP by the nucleotide sanitization enzyme MTH1, the nucleotide excision repair of DNA, and the apoptosis of oxidatively damaged cells $[18,19]$. Urinary excretion of 8 -OHdG reflects general average risk of promutagenic oxidative adducts in DNA, and thus carcinogenesis of all tissues and organs. Therefore, 8 -OHdG has become known as a useful biomarker of oxidative DNA damage $[20,21]$.

By the use of 8-OHdG as the biomarker, antioxidant activities of various nutrients such as carotenoids and vitamins have been evaluated [22]. However, limited clinical studies are available on the effective reduction of $8-\mathrm{OHdG}$ by the administration of CoQ10 so far. It seems to be due to the insufficient bioavailability of CoQ10 by oral supplementation requiring very high dose of CoQ10 to obtain significant results. In this study, we evaluated the antioxidant effects of CoQ10 on reducing 8-OHdG in urine by the oral intake of CoQ10 $\gamma \mathrm{CD}$ complex among addicted cigarette smokers having higher levels of urinary 8-OHdG than among non-smokers.

Test procedure: Urine samples, collected three times, before intake, after three weeks and after six weeks in the early morning just after wake up, were kept in tubes stored in a cooler box overnight and then frozen in chest freezer below $-5^{\circ} \mathrm{C}$ until analysis. Urinary samples were analyzed for 8-OHdG by HPLC.

\section{Study on protection of muscle cells by the evaluation of CPK and $\mathrm{LDH}$ in plasma and Creatinine in urine}

Oxidative stress plays an important role as a mediator of myocardial damage caused by bad living habits like smoking, alcohol drink and exhaustive exercise $[23,24]$. CoQ10 is known as a radical scavenger and antioxidant that is effective on recovery of cardiac function and also relieving fatigue [25]. There is one report on the muscle protection effect by CoQ10 supplementation on exhaustive exercise-induced muscular injury of rats by the evaluation of the concentration changes of enzyme biomarkers, creatinine phosphokinase (CPK) and lactate dehydrogenase (LDH) in plasma [26,27]. CPK and LDH enzyme activity is found in cardiac- and skeletal muscle cells and in blood. The elevation of serum CPK and LDH in plasma is caused through means of their tissue damage at least in part by oxidative stress [28]. Leakage of these cytosolic enzymes in plasma increased due to the change in the permeability of plasma membrane can result as a consequence of peroxidation of membrane by oxidants and free radicals. The protective ability of antioxidants may be due to its cell membrane stabilizing effect 
and radical scavenging potency.

Here, we evaluated the serum CPK enzyme activity level and LDH enzyme activity level in plasma by oral intake of CoQ10 - $\gamma$ CD complex as the absorbable form of CoQ10 as an antioxidant applied for addicted cigarette smokers having higher daily oxidative stress than nonsmokers. It is also known that creatinine $(\mathrm{Cr})$ is a biomarker excreted in urine by myocardial damage. The concentration changes of $\mathrm{Cr}$ level in plasma by the intake of CoQ10 - $\gamma \mathrm{CD}$ complex were also evaluated.

Test procedure: Blood samples were collected from the subjects in the morning of the day before intake, and after three weeks and six weeks after start of intake. $\mathrm{CPK}, \mathrm{LDH}$ and $\mathrm{Cr}$ in the blood samples were measured in accordance with the common procedure described by King [29] and Okinaka et al. [30].

\section{Results and discussion}

\section{Study on the curative effect on damaged human skins}

Wrinkles: Wrinkles at tail of eyes were evaluated by picture analysis (3D skin analysis: ASA-03R, Asashi-biomed Co., Ltd.). One example is shown in Figure 2, and the test results are summarized in Table 3. The colored part on the pictures helps to visualize the wrinkles. Comparing the colored pictures it's obvious that after intake of CoQ10 - $\gamma$ CD complex for six weeks wrinkles were decreased visually. Wrinkle volume, depth and width did not show significant difference. On the other hands, the number of wrinkle on the certain line (1 mm length) was significantly decreased. The result shows that the wrinkles on the skin were significantly diminished.

Textures: The skin textures were evaluated by picture analysis (3D skin analysis: ASA-03R, Asashi-biomed Co., Ltd.). The evaluation area was on the right cheek and $7 \mathrm{~cm}$ from the right earlobe on the line
Original pictures
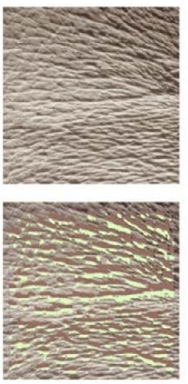

Before intake

Figure 2. Female (age: 44), tail of right eye.
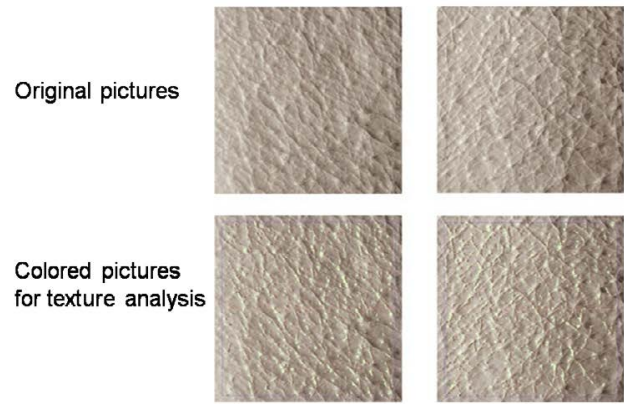

Before intake
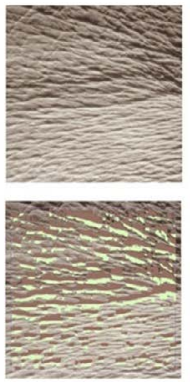

After 3 weeks

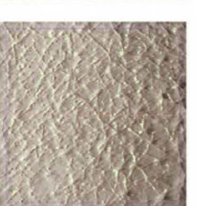

After 3 weeks
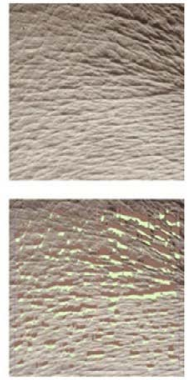

After 6 weeks
Figure 3. Female (age: 43), right cheek. between right wings of the nose and right earlobe. One example is shown in Figure 3 and the evaluation results are summarized in Table 4. The colored part of the pictures shows the texture on the skin. The depth of skin textures did not show significant difference, but the volume and number of texture significantly improved after intake for six weeks. The picture for analysis after intake for six weeks shows homogeneous texture distribution and significant larger area than before the intake. As a consequence, the skin texture conditions were significantly improved.

Skin elasticity and moisture content: Skin elasticity and moisture content were evaluated in the air conditioning room $\left(22^{\circ} \mathrm{C} \pm 1{ }^{\circ} \mathrm{C}\right.$, $\mathrm{RH} 50 \% \pm 10 \%)$ to avoid the impact on skin by different conditions in the atmosphere. The evaluation area of skin elasticity was on the right cheek and $4 \mathrm{~cm}$ from right earlobe on the line

between right edge of mouth and right earlobe by CUTOMETER MPA580 (Integral Corporation). The evaluation area of the moisture content were on right cheek and $5 \mathrm{~cm}, 5.5 \mathrm{~cm}$ and $6 \mathrm{~cm}$ (three areas) from right earlobe on the line between right edge of mouth and right earlobe by CORNEOMETER CM825 (Integral Corporation). The averaged values of moisture content from the three areas were calculated. The results are summarized in Table 5 and one representative skin macrophotograph example is shown in Figure 4. A significant increase of skin elasticity was observed after the intake for six weeks. This result clearly suggests that CoQ10 stimulates collagen production by human fibroblasts in corium. The moisture content of skin statistically did not show significant different data.

\section{Study on antioxidant effect by the evaluation of 8-OHdG in} urine

The levels of urinary 8-OHdG, a biomarker of oxidative DNA damage, were measured using a high-pressure liquid chromatography and normalized based on urinary creatinine levels before statistical analysis. The urinary 8-OHdG levels decreased significantly $(P<0.001)$ as shown in Figure 5. Not only $8-\mathrm{OHdG}$ levels but also creatinine levels to be generally used for the correction of 8-OHdG concentration constantly and significantly decreased. Since CoQ10 has also muscle protection effects which reflect to reduce creatinine levels, the general correction method of $8-\mathrm{OHdG}$ levels by creatinine levels might not be the right evaluation way for antioxidant activity of CoQ10 and we need to discuss basic methodologic issues. The creatinine level in urine and blood will be discussed in the next section. But even after correcting by creatinine levels, the ratio of $8-\mathrm{OHdG}$ levels/creatinine levels also significantly $(P=0.044)$ diminished after intake for six weeks as shown in Figure 6. Here, we could prove that the daily intake of CoQ10 with absorbable CoQ10 - $\gamma \mathrm{CD}$ complex reduces urinary 8-OHdG, suggesting the reduction of risk of cancer.

Study on protection of muscle cells by the evaluation of CPK and LDH in plasma and Creatinine in urine

The results of changes of CPK and LDH levels before and after intake of CoQ10 - $\gamma \mathrm{CD}$ complex are shown in Figure 8 and Figure 9.
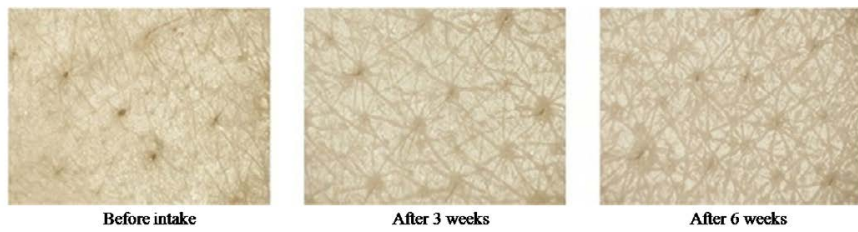

Figure 4. Macrophotograph of skin (Female, age:32, right cheek). 


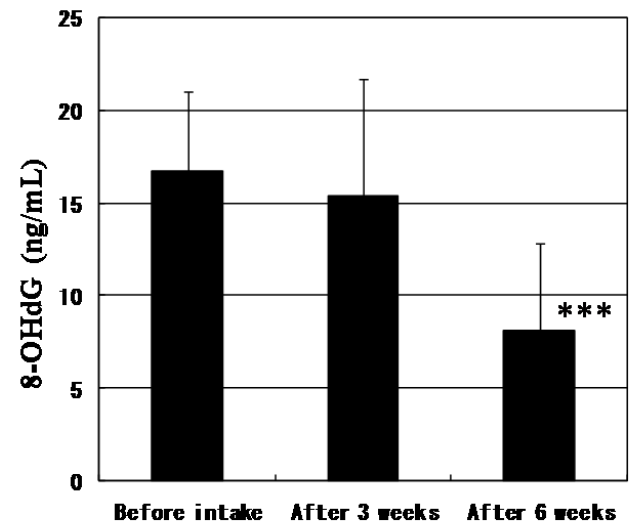

Statistical analysis was carried out by Dunnett method. $(n=18)$ (***: $P<0.001)$

Figure 5. Change of urinary $8-\mathrm{OHdG}$ levels.

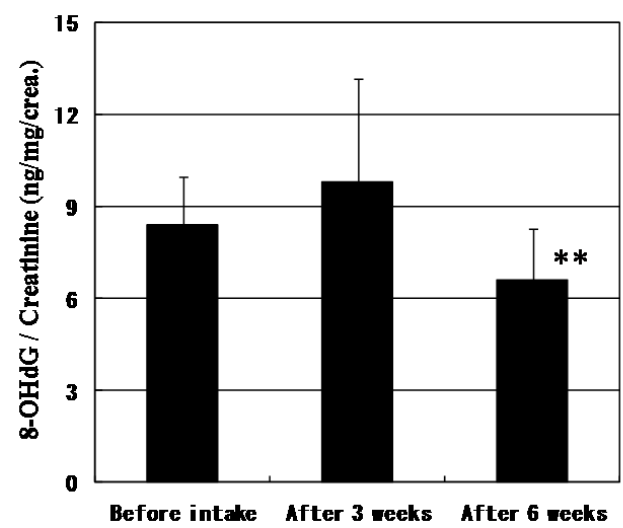

Statistical analysis was carried out by Dunnett method. $(n=18)$ $\left({ }^{* *}: P<0.01\right)$

Figure 6. Change of the ratio of urinary $8-\mathrm{OHdG} /$ creatinine levels.

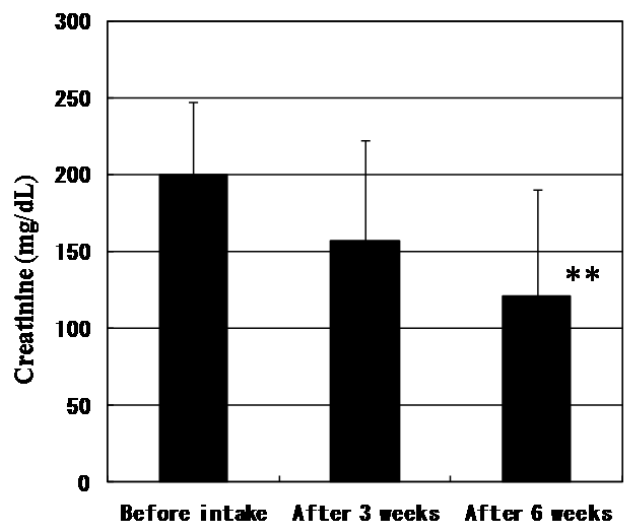

Statistical analysis was carried out by Dunnett method. $(n=18)$ $(\boldsymbol{*}: \mathbf{P}<\mathbf{0 . 0 1 )}$

Figure 7. Change of creatinine levels.

The mean value of CPK level in plasma of 18 subjects at $273.2 \mathrm{IU} / \mathrm{L}$ before intake decreased to $99.2 \mathrm{IU} / \mathrm{L}$ and $88.6 \mathrm{IU} / \mathrm{L}$ after intake for three and six weeks, respectively. According to the statistical analysis by the Dunnett method, the mean value of CPK level after intake for six weeks showed a significant difference compared to that before intake $(P<0.05)$. The mean level of LDH in plasma of 18 subjects at $167.6 \mathrm{IU} / \mathrm{L}$ before intake also decreased to $155.9 \mathrm{IU} / \mathrm{L}$ and $150.8 \mathrm{IU} / \mathrm{L}$ after intake for three and six weeks, respectively. Here again, the mean after intake for six weeks showed a significant difference compared to that before intake $(P<0.05)$. The decreased CPK and LDH levels after intake of CoQ10 - $\gamma \mathrm{CD}$ complex support the argument for the actions of CoQ10 as a radical scavenger and antioxidant protecting muscle cells.

Figure 10 shows the changes of $\mathrm{Cr}$ levels in plasma. The mean values of $\mathrm{Cr}$ levels in plasma of 18 subjects before intake and after intake for three and six weeks were at $0.70,0.68$ and $0.70 \mathrm{mg} / \mathrm{dL}$, respectively. Significant differences could not be found. On the other hand, the mean value of urinary $\mathrm{Cr}$ levels at $200.1 \mathrm{mg} / \mathrm{dL}$ before intake decreased to 157.1 and $120.9 \mathrm{mg} / \mathrm{dL}$ after intake for three and six weeks, respectively (Figure 7). There is a significant difference between before intake and after six weeks according to Dunnet test $(P<0.01)$. We reason that due to vital function maintaining homeostasis, $\mathrm{Cr}$ levels in plasma were kept to be same levels even under the exposure by oxidative stress. But we could observe the significant decrease of urinary $\mathrm{Cr}$ level by the intake of CoQ10 - $\gamma \mathrm{CD}$ complex that also shows the effectiveness of CoQ10 as an antioxidant for protection of muscle cells.

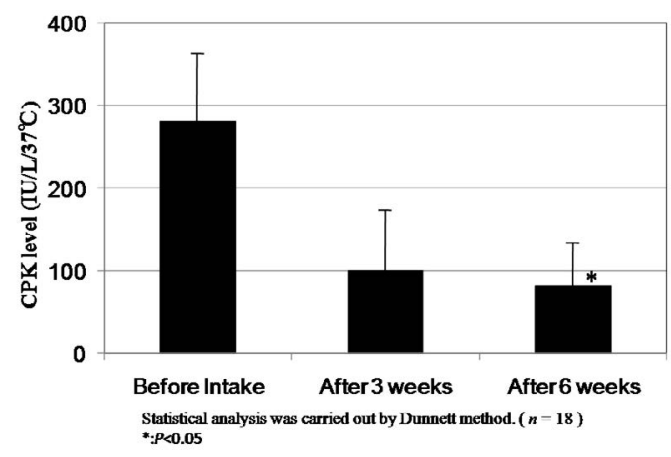

Figure 8. CPK levels in serum.

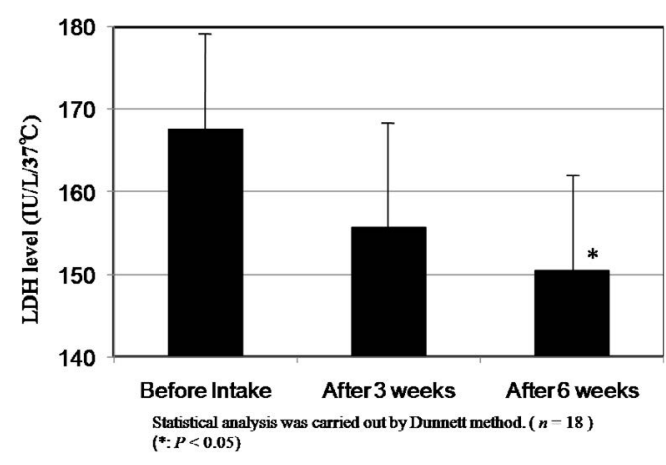

Figure 9. LDH levels in serum.

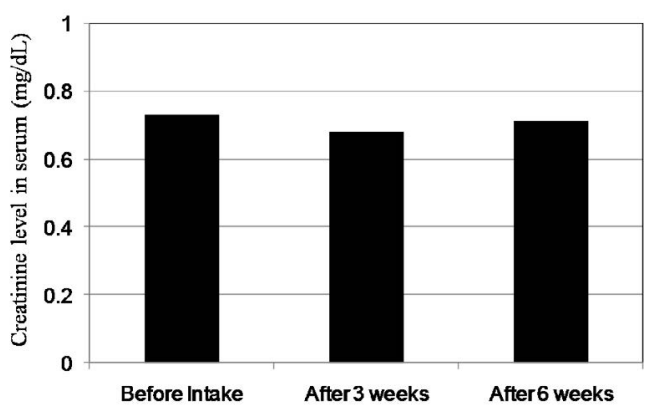

Figure 10. Creatinine levels in serum. 


\section{Conclusion}

There have been a dozen of articles reported with regard to various beneficial health effects by CoQ10 supplementation so far. But in most instances, significantly high doses of CoQ10 with more than $300 \mathrm{mg}$ per day have been required to obtain obvious health benefits, despite of long intensive argumentations for the adequate daily CoQ10 dose level. We have investigated the bioavailability enhancement of CoQ10 using CDs and successfully achieved long-lasting high plasma CoQ10 levels only by intake of a low dose of CoQ10 at $30 \mathrm{mg}$ per day as a CoQ10 $\gamma \mathrm{CD}$ complex.

Our next target was to confirm whether or not CoQ10 derived from CoQ10 - $\gamma \mathrm{CD}$ complex could adequately transfer from plasma to diverse tissues of the body. Since it is practically impossible to evaluate CoQ10 concentration in most tissues of living bodies, we evaluated the improvements of skin conditions, the reduction of 8-OHdG levels in urine as biomarker for DNA damage, the change of CPK and LDH levels in blood and $\mathrm{Cr}$ in urine to analyse the reduction of muscular injury by enhancing stabilization of muscle cell membrane. In conclusion, all results of the present investigations support that the long-lasting high plasma CoQ10 levels by the intake CoQ10 - $\gamma \mathrm{CD}$ complex could provide various valuable cell-protecting, anti-aging and health benefits of CoQ10.

\section{References}

1. Littarru GP, Tiano L (2007) Bioenergetic and antioxidant properties of coenzyme Q10: recent developments. Mol Biotechnol 37: 31-37. [Crossref]

2. Kumar A, Kaur H, Devi P, Mohan V (2009) Role of coenzyme Q10 (CoQ10) in cardiac disease, hypertension and Meniere-like syndrome. Pharmacol Ther 124: 259-268. [Crossref]

3. Dahan A, Beig A, Lindley D, Miller JM (2016) The solubility-permeability interplay and oral drug formulation design: Two heads are better than one. Adv Drug Deliv Rev 101: 99-107. [Crossref]

4. Savjani KT, Gajjar AK, Savjani JK (2012) Drug solubility: importance and enhancement techniques. ISRN Pharm 2012: 195727. [Crossref]

5. Del Valle EMM (2004) Cyclodextrins and their uses: a review. Proc Biochem 39: 10331046

6. Loftsson T, Duchêne D (2007) Cyclodextrins and their pharmaceutical applications. Int J Pharm 329: 1-11. [Crossref]

7. Terao K, Nakata D, Fukumi H, Schmid G, Arima H, et al. (2006) Enhancement of oral bioavailability of coenzyme Q10 by complexation with ?-cyclodextrin in healthy adults. Nutrition Research 26: 503-508.

8. Gao X, Nishimura K, Hirayama F, Arima H, Uekama K, et al. (2006) Enhanced dissolution and oral bioavailability of coenzyme Q10 in dogs obtained by inclusion complexation with $\gamma$-cyclodextrin. Asian Journal of Pharmaceutical Sciences 1: 95102 .

9. Terao K, Konishi M, Nakata D, Uekaji Y (2005) Long known but brand new useable tool for food science -New use of natural cyclodextrins. Food Ingredients Journal of Japan 201: 222-243.

10. Terao K (2007) Bioavailability enhancement of coenzyme Q10 by complexation with $\gamma$-cyclodextrin. International Life Sciences Institute of Japan 90: 15-27.
11. Nakata D, Nakano M, Terao K (2007) Bioavailability enhancement of coenzyme Q10 by use of gamma cyclodextrin. 5th Conference of the International Coenzyme Q10 Association. Abstract 172-173.

12. Morita A (2007) Tobacco smoke causes premature skin aging. J Dermatol Sci 48: 169175. [Crossref]

13. Udompataikul M, Sripiroj P, Palungwachira P. (2009) An oral nutraceutical containing antioxidants, minerals and glycosaminoglycans improves skin roughness and fine wrinkles. Int J Cosmet Sci 31: 427-435.

14. Hoppe U, Bergemann J, Diembeck W, Ennen J, Gohla S, et al. (1999) Coenzyme Q10, a cutaneous antioxidant and energizer. Biofactors 9: 371-378. [Crossref]

15. Gerhardt LC, Lenz A, Spencer ND, Münzer T, Derler S (2009) Skin-textile friction and skin elasticity in young and aged persons. Skin Res Technol 15: 288-298. [Crossref]

16. Krinsky NI (2000) Panel on Dietary Antioxidants and Related Compounds. In: Dietary reference intakes for vitamin C, vitamin E, selenium and carotenoids. National Academy Press, Washington, D.C.

17. Ames BN (1989) Endogenous oxidative DNA damage, aging, and cancer. Free Radic Res Commun 7: 121-128. [Crossref]

18. Loft S, Vistisen K, Ewertz M, Tjønneland A, Overvad K, et al. (1992) Oxidative DNA damage estimated by 8-hydroxydeoxyguanosine excretion in humans: influence of smoking, gender and body mass index. Carcinogenesis 13: 2241-2247. [Crossref]

19. Kasai H, Iwamoto-Tanaka N, Miyamoto T, Kawanami K, Kawanami S, et al. (2001) Life style and urinary 8-hydroxydeoxyguanosine, a marker of oxidative dna damage: effects of exercise, working conditions, meat intake, body mass index, and smoking. Jpn J Cancer Res 92: 9-15. [Crossref]

20. Loft S, Poulsen HE (1996) Cancer risk and oxidative DNA damage in man. J Mol Med (Berl) 74: 297-312. [Crossref]

21. Cooke MS, Evans MD, Herbert KE, Lunec J (2000) Urinary 8-oxo-2'-deoxyguanosine-source, significance and supplements. Free Radic Res 32: 381-397. [Crossref]

22. Cocate PG, Natali AJ, Alfenas RC, de Oliveira A, dos Santos EC, et al. (2015) Carotenoid consumption is related to lower lipid oxidation and DNA damage in middle-aged men. Br J Nutr 114: 257-264. [Crossref]

23. Farsalinos K, Tsiapras D, Kyrzopoulos S, Voudris V (2013) Acute and chronic effects of smoking on myocardial function in healthy heavy smokers: a study of Doppler flow, Doppler tissue velocity, and two-dimensional speckle tracking echocardiography. Echocardiography 30: 285-292. [Crossref]

24. O'Keefe JH, Patil HR, Lavie CJ, Magalski A, Vogel RA, et al. (2012) Potential adverse cardiovascular effects from excessive endurance exercise. Mayo Clin Proc 87: 587595. [Crossref]

25. Ayer A, Macdonald P, Stocker R (2015) CoQâ, â,€ Function and Role in Heart Failure and Ischemic Heart Disease. Annu Rev Nutr 35: 175-213. [Crossref]

26. Kon M, Kimura F, Akimoto T, Tanabe K, Murase Y, et al. (2007) Effect of Coenzyme Q10 supplementation on exercise-induced muscular injury of rats. Exerc Immunol Rev 13: 76-88. [Crossref]

27. Adams JE 3rd, Miracle VA (1998) Cardiac biomarkers: past, present, and future. Am J Crit Care 7: 418-423. [Crossref]

28. Narayanan S, Appleton HD (1980) Creatinine: a review. Clin Chem 26: 1119-1126 [Crossref]

29. King J (1965) The dehydrogenases or oxidoreductase-lactate dehydrogenase. In: Practical Clinical Enzymology. Nostrand, London, 83-93.

30. Okinaka S, Kumagai H, Ebashi S, Sugita H, Momoi H, et al. (1961) Serum creatine phosphokinase. Activity in progressive muscular dystrophy and neuromuscular diseases. Arch Neurol 4: 520-525. [Crossref]

Copyright: (C2017 Uekaji Y. This is an open-access article distributed under the terms of the Creative Commons Attribution License, which permits unrestricted use, distribution, and reproduction in any medium, provided the original author and source are credited. 